Research Article

\title{
Florfenicol Resistance in Enterobacteriaceae and Whole-Genome Sequence Analysis of Florfenicol-Resistant Leclercia adecarboxylata Strain R25
}

\author{
Yuanyuan Ying $\mathbb{D}^{1},{ }^{1}$ Fei Wu, ${ }^{1}$ Chongyang Wu, ${ }^{1}$ Yi Jiang, ${ }^{1}$ Min Yin, ${ }^{1}$ Wangxiao Zhou, \\ Xinyi Zhu, ${ }^{1}$ Cong Cheng $\left({ }^{\circ},{ }^{2}\right.$ Licheng $\mathrm{Zhu},{ }^{2}$ Kewei Li, ${ }^{1}$ Junwan $\mathrm{Lu}\left({ }^{1},{ }^{1,2}\right.$ Teng $\mathrm{Xu},{ }^{3}$ \\ and Qiyu Bao $\mathbb{1}^{1}$ \\ ${ }^{1}$ School of Laboratory Medicine and Life Sciences/Institute of Biomedical Informatics, Wenzhou Medical University, Wenzhou, \\ Zhejiang 325035, China \\ ${ }^{2}$ College of Medicine and Health, Lishui University, Lishui 323000, China \\ ${ }^{3}$ Institute of Translational Medicine, Baotou Central Hospital, Baotou 014040, China
}

Correspondence should be addressed to Qiyu Bao; ym888888ym@163.com

Received 20 March 2019; Accepted 30 August 2019; Published 1 October 2019

Academic Editor: Paulo M. Pinto

Copyright ( 2019 Yuanyuan Ying et al. This is an open access article distributed under the Creative Commons Attribution License, which permits unrestricted use, distribution, and reproduction in any medium, provided the original work is properly cited.

\begin{abstract}
Due to inappropriate use, florfenicol resistance is becoming increasingly serious among animal respiratory tract and gut bacteria. To detect the florfenicol resistance mechanism among Enterobacteriaceae bacteria, 292 isolates from animal feces were examined. The agar dilution method was conducted to determine the minimum inhibitory concentration (MIC) for florfenicol, and polymerase chain reaction (PCR) was performed to detect florfenicol resistance genes. To further explore the molecular mechanism of florfenicol resistance, the whole-genome Leclercia adecarboxylata R25 was sequenced. Of the strains tested, $61.6 \%$ $(180 / 292)$ were resistant to florfenicol, $64.4 \%$ (188/292) were positive for $f l o R$, and $1.0 \%(3 / 292)$ for $c f r$. The whole-genome sequence analysis of $L$. adecarboxylata R25 revealed that the floR gene is carried by a transposon and located on a plasmid (pLA-64). Seven other resistance genes are also encoded on pLA-64, all of which were found to be related to mobile genetic elements. The sequences sharing the greatest similarities to pLA-64 are the plasmids p02085-tetA of Citrobacter freundii and p234 and p388, both from Enterobacter cloacae. The resistance gene-related mobile genetic elements also share homologous sequences from different species or genera of bacteria. These findings indicate that floR mainly contributes to the high rate of florfenicol resistance among Enterobacteriaceae. The resistance gene-related mobile genetic elements encoded by pLA-64 may be transferred among bacteria of different species or genera, resulting in resistance dissemination.
\end{abstract}

\section{Introduction}

Enterobacteriaceae bacteria are important species that comprise the gut microbiota of domestic animals. There are reports that members of this family cause infections, for example, Salmonella Gallinarum as the cause of septicemia in fowl typhoid, Escherichia coli causing severe respiratory diseases in poultry and bovine mastitis, and septicemia in pigs due to Klebsiella pneumoniae. Overall, the uncontrolled use of antibiotics for the treatment and prevention of infectious diseases in animals as well as their application as growth promoters in animal husbandry has contributed to the increased spread of antimicrobial resistance genes among Enterobacteriaceae bacteria, resulting in significant economic losses [1].

Florfenicol, a synthetic broad-spectrum antibiotic derived from chloramphenicol but with better antibacterial activity and few adverse effects, has been universally used in veterinary medicine $[2,3]$. However, due to inappropriate use to prevent or cure bacterial infections, florfenicol resistance has become increasingly serious and a variety of florfenicol resistance mechanisms have been characterized, including efflux pumps, rRNA methyltransferases, and chloramphenicol acetate esterases. To 
TABLE 1: Bacterial strains and plasmids used in this study.

\begin{tabular}{|c|c|c|}
\hline Strains and plasmids & Description & Source \\
\hline \multicolumn{3}{|l|}{ Strain } \\
\hline L. adecarboxylata R25 & Multiresistant isolate derived from rabbits & This study \\
\hline E. coli $\mathrm{DH} 5 \alpha$ & Used as a host for cloning of PCR products & Our lab collection \\
\hline E. coli ATCC25922 & Used as a control strain & Our lab collection \\
\hline $\mathrm{pMD}^{\mathrm{TM}} 19-\mathrm{T}-\mathrm{ORF} / E$. coli $\mathrm{DH} 5 \alpha$ & $\begin{array}{l}\text { E. coli } \mathrm{DH} 5 \alpha \text { carrying the recombinant plasmid } \\
\text { pMD }{ }^{\mathrm{TM}} 19-\mathrm{T} \text { carrying resistance gene ORFs and } \\
\text { promoter regions }\left(a a c\left(6^{\prime}\right)-I b-c r, \text { aadA16, arr-3, qnrB6, and floR) }\right.\end{array}$ & This study \\
\hline \multicolumn{3}{|c|}{$x^{2}$} \\
\hline $\mathrm{pMD}^{\mathrm{TM}} 19-\mathrm{T}$ & $\begin{array}{l}\text { Cloning vector for the PCR products of all resistance } \\
\text { genes and its promoter region, } \mathrm{Amp}^{\mathrm{r}}\end{array}$ & This study \\
\hline
\end{tabular}

Abbreviations: Amp: ampicillin; r: resistance.

date, seven florfenicol resistance genes, floR, $c f r, f e x A, f e x B$, pexA, optrA, and estDL136 [3-6], together with some variants $(f l o R v, f l o S t, c f r(\mathrm{~B})$, and $c f r(\mathrm{C}))$ [7-9] have been discovered. Nonetheless, only a limited number of studies have reported the resistance to florfenicol or distribution of florfenicol resistance genes among Enterobacteriaceae, mainly including common species such as Escherichia coli, K. pneumoniae, Salmonella enterica, Yersinia enterocolitica, and Proteus vulgaris [10-14]. Moreover, the florfenicol resistance mechanisms of most Enterobacteriaceae species have not been investigated.

The genus Leclercia of the family Enterobacteriaceae contains only one species, $L$. adecarboxylata, which is a characteristic of a gram-negative, motile, and facultative anaerobic bacillus. First described as Escherichia adecarboxylata by Leclerc in 1962 [15], the species was renamed L. adecarboxylata by Tamura et al. in 1986 according to the recognition of its phenotypic and genotypic differences from species of the genus Escherichia and other species of Enterobacteriaceae [16]. L. adecarboxylata, which is normally present in environmental or animal sources [17], is an opportunistic human pathogen but is rarely isolated from clinical specimens. Nonetheless, it has been reported to cause bacteremia, sepsis, peritonitis, cellulitis, endocarditis, and cholecystitis in immunocompromised patients with polymicrobial infections [18]. There is no report thus far of a florfenicol molecular resistance mechanism of $L$. adecarboxylata isolated from an animal or the environment. In this work, we analyzed florfenicol resistance and the resistance genes of animal-derived Enterobacteriaceae bacteria and further examined L. adecarboxylata strain R25 to demonstrate the molecular resistance mechanism against florfenicol of this unique Enterobacteriaceae species.

\section{Materials and Methods}

2.1. Bacterial Strain and PCR Detection of Florfenicol Resistance-Associated Genes. Enterobacteriaceae strains were isolated from anal fecal samples obtained on food animalproducing farms (ducks, chickens, cows, geese, and rabbits) in Wenzhou, Zhejiang Province, China, from July 2014 to November 2015. A total of 292 Enterobacteriaceae strains were isolated. Species identification was conducted using a bioMérieux VITEK ${ }^{\circledR} 2$ Compact Instrument (bioMérieux,
Marcy L'etoile, France) and comparative analysis of $16 \mathrm{~S}$ rRNA gene sequences from bacteria of the same genera in the National Center for Biotechnology Information (NCBI) database (https://blast.ncbi.nlm.nih.gov/Blast.cgi). Further verification of $L$. adecarboxylata R25 was conducted by homologous comparisons of the whole-genome sequences with those in NCBI. The bacterial strains and plasmids used in this study are listed in Table 1.

Bacterial genomic DNA was extracted using an AxyPrep Bacterial Genomic DNA Miniprep Kit (Axygen Scientific, Union City, CA, USA) and used as the template for subsequent PCR. PCR amplification was conducted to screen florfenicol resistance genes $f l o R, c f r, \operatorname{pex} A, f e x A, f e x B$, and estDL136. Primers were designed by using Primer Premier 5.0 (Table 2). The PCR products were further confirmed by Sanger sequencing (ABI 3730 Analyzer, Foster City, CA, USA). Both strands of the PCR products were sequenced with the forward and reverse primers, and the sequencing reads were assembled with the Phred/Phrap/Consed software package (http://www.phrap.org/phredphrapconsed.html). The sequence data were compared to the NCBI nucleotide sequence database using BLAST with the max target sequences of 100 , expect threshold of 10 , word size of 28 , max matches in a query range of 0 , match scores of 1 , and mismatch scores of -2 (https://blast.ncbi.nlm.nih.gov).

2.2. Antibiotic Susceptibility Assay. The MICs of antimicrobial agents against the 292 Enterobacteriaceae strains and corresponding recombinants carrying cloned resistance genes were determined using the standard agar dilution method recommended by the Clinical and Laboratory Standards Institute (CLSI document M100-S27, 2017). A bacterial suspension was adjusted to a turbidity equivalent to a $0.5 \mathrm{McF}$ arland standard with sterilized saline solution (0.9\%) and plated on MuellerHinton agar containing different concentrations of various antimicrobial agents. The plates were incubated at $37^{\circ} \mathrm{C}$ for $24 \mathrm{~h}$. The MIC was recognized as the lowest antibiotic concentration resulting in no colony growth. Each of the tests was carried out in triplicate. E. coli ATCC 25922 was used as a quality control strain. The resistance breakpoints of Enterobacteriaceae for florfenicol were set referring to those for chloramphenicol in the guidelines of CLSI document M100-S27 (2017). 
TABLe 2: Primers used in this work.

\begin{tabular}{|c|c|c|c|c|}
\hline Primer & Sequence $\left(5^{\prime}-3^{\prime}\right)$ & Purpose & $\begin{array}{c}\text { Product } \\
\text { length (bp) }\end{array}$ & $\begin{array}{c}\text { Annealing } \\
\text { temperature }\left({ }^{\circ} \mathrm{C}\right)\end{array}$ \\
\hline $27 \mathrm{~F}$ & AGAGTTTGATCCTGGCTCAG & \multirow{2}{*}{$16 \mathrm{~S}$ rRNA } & \multirow{2}{*}{1465} & \multirow{2}{*}{55} \\
\hline $1492 \mathrm{R}$ & TACGGCTACCTTGTTACGACTT & & & \\
\hline floR-F & ATGGTGATGCTCGGCGTGGGCCA & \multirow{2}{*}{ floR gene screening } & \multirow{2}{*}{800} & \multirow{2}{*}{58} \\
\hline floR-R & GCGCCGTTGGCGGTAACAGACACCGTGA & & & \\
\hline$c f r-\mathrm{F}$ & GGGAGGATTTAATAAATAATTTTGGAGAAACAG & \multirow{2}{*}{$c f r$ gene screening } & \multirow{2}{*}{580} & \multirow{2}{*}{58} \\
\hline$c f r-\mathrm{R}$ & CTTATATGTTCATCGAGTATATTCATTACCTCATC & & & \\
\hline pexA-F & CTTCCAGTTGAGAAGCGAGC & \multirow{2}{*}{ pexA gene screening } & \multirow{2}{*}{319} & \multirow{2}{*}{56} \\
\hline pexA-R & AGAAGCATACCCGTGAACATG & & & \\
\hline fexA-F & CTCTTCTGGACAGGCTGGAA & \multirow{2}{*}{ fexA gene screening } & \multirow{2}{*}{332} & \multirow{2}{*}{57} \\
\hline fexA-R & CCAGTTCCTGCTCCAAGGTA & & & \\
\hline fexB-F & ACTGGACAGGCAGGCTTAAT & \multirow{2}{*}{ fexB gene screening } & \multirow{2}{*}{319} & \multirow{2}{*}{57} \\
\hline fexB-R & CCTGCCCCAAGATACATTGC & & & \\
\hline optrA-F & CTTATGGATGGTGTGGCAGC & \multirow{2}{*}{ optrA gene screening } & \multirow{2}{*}{309} & \multirow{2}{*}{56} \\
\hline optrA-R & CCATGTGGTTTGTCGGTTCA & & & \\
\hline estDL136-F & ATGCCGTTAAACCCCCATGTCGAAG & \multirow{2}{*}{ estDL136 gene screening } & \multirow{2}{*}{933} & \multirow{2}{*}{55} \\
\hline estDL136-R & TCAAGCGAGGTCTCTTTTAAGATT & & & \\
\hline pro- $a a c\left(6^{\prime}\right)-I b-c r-\mathrm{F}$ & GCTATCAGGTCAAGTCTGCTTTTATT & \multirow{2}{*}{$a a c\left(6^{\prime}\right)-I b-c r$ gene cloning } & \multirow{2}{*}{674} & \multirow{2}{*}{62} \\
\hline pro- $a a c\left(6^{\prime}\right)-I b-c r-\mathrm{R}$ & TTAGGCATCACTGCGTGTTCGCTCG & & & \\
\hline pro-aadA16-F & GTGTTTCCATCTATAGAAGCAGCAATG & \multirow{2}{*}{ aadA16 gene cloning } & \multirow{2}{*}{1274} & \multirow{2}{*}{56} \\
\hline pro-aadA16-R & TTAAGCTGCGCCGCGAAGCGGCGTC & & & \\
\hline pro-arr-3-F & GGTGACCAACAGCAACGATTCCGTCAC & \multirow{2}{*}{ arr-3 gene cloning } & \multirow{2}{*}{1104} & \multirow{2}{*}{62} \\
\hline pro-arr-3-R & CTAGTCTTCAATGACGTGTAAACCAC & & & \\
\hline pro-qnrB6-F & GTTATTATGCACGGCTTACAGCAGGCAA & ane 16 rene sloning & & \\
\hline pro-qnrB6-R & CTAACCAATCACCGCGATGCCAAGCCG & qnrB6 gene cloning & 849 & 62 \\
\hline pro-floR-F & GTTGCGAAGCAAAAGATAATCGGATAAA & & & \\
\hline pro-floR-R & TTAGACGACTGGCGACTTCTCGGTGGCA & ng & 1415 & 62 \\
\hline
\end{tabular}

2.3. Whole-Genome Sequencing. A $20 \mathrm{~kb}$ library was generated using a SMRTbell Template Prep Kit (Pacific Biosciences, Menlo Park, CA, United States) according to the PacBio standard protocol and sequenced using a PacBio RS II instrument. In addition, an Illumina library with $300 \mathrm{bp}$ insert sizes was constructed and sequenced from both ends using the HiSeq 2500 platform (both PacBio RS II and HiSeq 2500 sequencing were carried out at Annoroad Gene Technology Co. Ltd., Beijing, China). Reads of the clean data derived from the raw data of HiSeq 2500 sequencing were initially assembled de novo with the SOAPdenovo software to obtain contigs of the genome sequences. PacBio long reads were assembled using Canu software [19]. Two FASTQ sequence files corresponding to the reads derived from HiSeq 2500 sequencing were used to control assembly quality and to correct possibly misidentified bases. Potential ORFs were predicted using Glimmer software (http://ccb.jhu.edu/software.shtml) and annotated against a nonredundant protein database using BLASTX (https://blast.ncbi.nlm.nih.gov). Plasmid typing was performed using BLAST in the PlasmidFinder database (https://cge.cbs.dtu.dk/services/MLST/).
2.4. Cloning of Resistance Genes. The primers used to clone candidate genes with potential upstream promoter regions are shown in Table 2. PrimeSTAR HS DNA Polymerase (TaKaRa, Dalian, China) was used to amplify resistance genes according to the manufacturer's instructions. A poly(A) tail was added to each purified PCR product (prom-ORF) using the DNA A-Tailing Kit (TaKaRa, Dalian, China), and the fragment was then cloned into the $\mathrm{pMD}^{\mathrm{TM}} 19-\mathrm{T}$ vector (TaKaRa, Dalian, China). The resulting recombinant plasmid (pMD ${ }^{\mathrm{TM}} 19-\mathrm{T}$-prom-ORF) was transformed into E. coli $\mathrm{DH} 5 \alpha$ using the calcium chloride method. Transformants were selected on LB agar plates containing $100 \mu \mathrm{g} / \mathrm{mL}$ ampicillin. The cloned PCR product was further confirmed by Sanger sequencing.

2.5. Comparative Genomic Analysis. The plasmid and chromosome genome sequences used in this study were downloaded from NCBI (http://www.ncbi.nlm.nih.gov). Comparisons of nucleotide and amino acid sequences were performed using BLASTN and BLASTP, respectively. The map of the plasmid with GC content and GC skew was drawn using the online CGView Server (http://stothard 
.afns.ualberta.ca/cgview_server/) and local GView 1.7 with visual interface [20]. Family classification and domain prediction of mobile genetic elements (MGEs) were verified by comparison using the InterPro database (http:// www.ebi.ac.uk/interpro/) and ISfinder database (https:// isfinder.biotoul.fr/). Other bioinformatics tools were utilized through Perl and BioPerl (http://www.perl.org/).

\section{Results and Discussion}

3.1. Animal Enterobacteriaceae Bacteria and Their Resistance to Phenicols. Florfenicol as well as tetracyclines, beta-lactams, and trimethoprim/sulfonamides is widely used to treat animal infections. The resistance rates for these antibiotics have increased greatly, and the emergence of multidrug-resistant bacteria is increasing [2]. In this work, we detected resistance to florfenicol and chloramphenicol among 292 Enterobacteriaceae strains isolated from fecal specimens of 5 types of animals (rabbit, chicken, cow, goose, and duck). The strains isolated belong to 11 genera: Escherichia (86.0\%, 251/292), Shigella (0.7\%, 2/292), Klebsiella (2.7\%, 8/292), Serratia (0.3\%, 1/292), Proteus (3.1\%, 9/292), Citrobacter (1.7\%, 5/292), Enterobacter (3.4\%, 10/292), Yersinia (0.3\%, 1/292), Leclercia (0.3\%, 1/292), Pantoea (1.0\%, 3/292), and Kluyvera $(0.3 \%, 1 / 292)$ (Table S1). The overall resistance rates to florfenicol and chloramphenicol were $61.6 \%$ and $65.1 \%$, respectively. Except for the 10 strains of Enterobacter, which exhibited low resistance rates of $20.0 \%$ to both florfenicol and chloramphenicol, all the other strains from various genera showed high resistance rates of 50.0-88.9\% to florfenicol and $64.1-100.0 \%$ to chloramphenicol. Proteus spp. exhibited the highest resistance rate of $88.9 \%(8 / 9)$ to florfenicol; Klebsiella spp. and Escherichia spp. also displayed high resistance rates of $75.0 \%(6 / 8)$ and $62.5 \%$ (157/251) to florfenicol, respectively (Table 3).

The rates of resistance to florfenicol among the Enterobacteriaceae bacteria isolated in this work appeared to be much higher than those reported for bacteria from the same or different genera or families. For example, in one study, isolates of Pasteurella multocida, Actinobacillus pleuropneumoniae, and Streptococcus suis from cattle and pig respiratory tract infections showed resistance rates to florfenicol of $<1 \%$ [21]. Another report demonstrated that the rates of florfenicol resistance of $A$. pleuropneumoniae and $P$. multocida isolated from pig respiratory tract infections were $2.0 \%$ and $6.0 \%$, respectively [22]. Resistance rates of $25.4 \%$ and $15.3 \%$ to florfenicol have also been reported for Salmonella and Yersinia, respectively $[13,23]$. In addition, E. coli strains from canine urinary tract infections showed higher resistance, at a rate of $31.6 \%(36 / 114)$, to florfenicol than did other pathogens [10]. More recently, a resistance level to chloramphenicol for Staphylococcus pseudintermedius of $32 \mu \mathrm{g} / \mathrm{mL}$ (MIC90) was reported, but no resistance to florfenicol was detected in this species (MIC90 $=4 \mu \mathrm{g} / \mathrm{mL}$ ) [24]. Despite these publications, there is no report thus far regarding the resistance to florfenicol for other species of Enterobacteriaceae. In this work, except for four genera with only 1 (Yersinia, Serratia, and Kluyvera) or 3 (Pantoea) isolates that were sensitive to florfenicol, the other 7 genera tested exhibited some degree of resistance to florfenicol. In particular, this is the first report of resistance to florfenicol among isolates of Shigella (1/2), Klebsiella (6/8), Proteus (8/9), Citrobacter (5/5), Enterobacter (2/10), and Leclercia $(1 / 1)$ isolated from animals.

3.2. Distribution of Florfenicol Resistance Genes among Animal Enterobacteriaceae Isolates. Seven florfenicol resistance genes (floR, fexA, fexB, pexA, cfr, optrA, and estDL136) have been identified among bacteria. Our PCR screening of florfenicol resistance genes among 292 Enterobacteriaceae isolates revealed positive results for only floR and $c f r$. Among the strains, 64.4\% (188/292) were positive for $f l o R$, whereas $c f r$ was only identified in three Proteus strains $(1.0 \%, 3 / 292)$ isolated from geese. No isolates were positive for pexA, fexA, fexB, optrA, or estDL136 (Table 3). With the exception of Enterobacter isolates exhibiting a low rate of positivity for the floR gene $(30.0 \%)$, the other genera showed high floR-positive rates of $50.0-88.9 \%$, in accordance with the florfenicol resistance rates within each genus. Proteus spp. exhibited the highest rates of resistance gene positivity, at $88.9 \%$ (8/9) for floR and $33.3 \%(3 / 9)$ for both floR and $c f r$. Of the seven florfenicol resistance genes, floR is the main and most common florfenicol resistance gene identified in both gram-positive and gram-negative bacteria [25] and the only one identified in $K$. pneumoniae strains originating from both humans and animals $[11,26]$. In our study, the rate of floR positivity for K. pneumonia was $75.0 \%$ (6/8), much higher than the $7.0 \%(23 / 328)$ of human clinical K. pneumonia isolated from the same district [11] and indicating the wide use of florfenicol in local animal farming.

The rates of floR gene positivity from various bacteria differed significantly. A high rate of floR gene positivity $(81.3 \%)$ has been reported for clinical Vibrio cholerae isolates from some Iranian provinces [27]. Regarding Salmonella isolates from broiler farms in East China, the overall rate of floR gene positivity was $43.5 \%$, and it is interesting that the rates between serotypes differed greatly. Salmonella enterica serovar Indiana isolates displayed a positive rate up to $96.2 \%$ (128/133), though that of S. enterica serovar Enteritidis strains was only $3.9 \%$ (7/177) [12]. Among gramnegative bacteria, the $c f r$ genes have been found in $P$. vulgaris (as in this work) and in E. coli $[6,14]$. Moreover, floR $v$ and floSt, variants of floR, have only been identified in a few gram-negative bacteria, including Stenotrophomonas maltophilia [8] and Salmonella [7], respectively. In contrast, estDL136 has only been identified in E. coli [6]. fexA, fexB, pexA, optrA, and $c f r$ were mainly harbored by gram-positive bacteria $[4,5,28]$, though fexA and pexA have been found in E. coli [6].

3.3. Sequencing Analysis of the L. adecarboxylata $R 25$ Genome. The floR gene has been found on the chromosome as well as plasmids. It was first identified on the chromosome of Salmonella typhimurium DT104 [29] and later on a plasmid of E. coli isolate BN10660 [30] and IncC plasmid R55 of K. pneumoniae [26], among others. However, no publication has reported the resistance level 


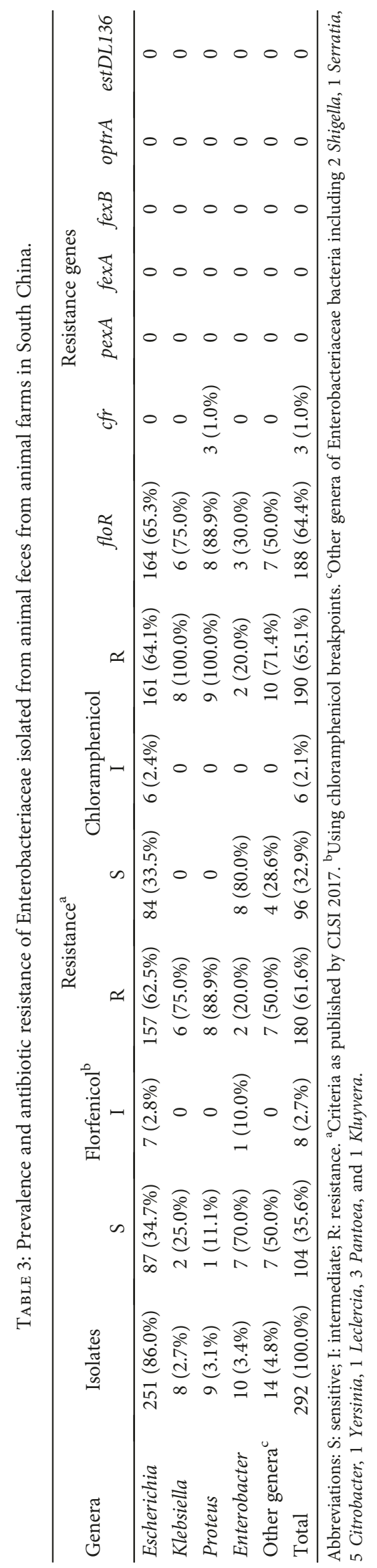


TABLe 4: MICs of antibiotics for the L. adecarboxylata R25 strain and its derivatives $(\mu \mathrm{g} / \mathrm{mL})$.

\begin{tabular}{|c|c|c|c|c|c|c|c|c|c|c|c|c|}
\hline Strain & FFC & $\mathrm{CHL}$ & RIF & AMK & GEN & STR & SPE & KAN & NEO & NAL & NOR & CIP \\
\hline L. adecarboxylata R25 & 128 & 128 & 256 & 2 & 0.125 & 8 & 64 & 4 & 0.5 & 8 & 1 & 0.25 \\
\hline $\mathrm{pMD}^{\mathrm{TM}} 19-\mathrm{T}-a a c\left(6^{\prime}\right)-I b-c r / E$. coli $\mathrm{DH} 5 \alpha$ & 8 & 8 & 16 & 16 & 0.25 & 4 & 8 & 64 & 2 & 4 & 0.13 & $<0.03$ \\
\hline $\mathrm{pMD}^{\mathrm{TM}} 19-\mathrm{T}$-aadA16/E. coli $\mathrm{DH} 5 \alpha$ & 8 & 8 & 16 & 2 & 0.25 & $>64$ & $>64$ & 1 & 2 & 4 & $<0.03$ & $<0.03$ \\
\hline pMD ${ }^{\mathrm{TM}} 19-\mathrm{T}-$ arr-3/E. coli $\mathrm{DH} 5 \alpha$ & 8 & 8 & 512 & 2 & 0.25 & 4 & 8 & 1 & 2 & 4 & $<0.03$ & $<0.03$ \\
\hline $\mathrm{pMD}^{\mathrm{TM}} 19-\mathrm{T}-q n r B 6 /$ E. coli $\mathrm{DH} 5 \alpha$ & 8 & 8 & 16 & 2 & 0.25 & 4 & 8 & 1 & 2 & 32 & 0.25 & 0.25 \\
\hline $\mathrm{pMD}^{\mathrm{TM}} 19-\mathrm{T}-$ floR/E. coli $\mathrm{DH} 5 \alpha$ & 64 & 32 & 16 & 2 & 0.25 & 4 & 8 & 2 & 2 & 4 & $<0.03$ & $<0.03$ \\
\hline E. coli $\mathrm{DH} 5 \alpha$ & 8 & 8 & 32 & 2 & 0.25 & 4 & 8 & 1 & 2 & 4 & 0.06 & $<0.03$ \\
\hline E. coli ATCC25922 & 4 & 4 & 8 & 4 & 0.5 & 8 & 8 & 4 & 2 & 2 & $<0.03$ & $<0.03$ \\
\hline
\end{tabular}

Abbreviations: FFC: florfenicol; CHL: chloramphenicol; RIF: rifampin; AMK: amikacin; GEN: gentamicin; STR: streptomycin; SPE: spectinomycin; KAN: kanamycin; NEO: neomycin; NAL: nalidixic acid; NOR: norfloxacin; CIP: ciprofloxacin.

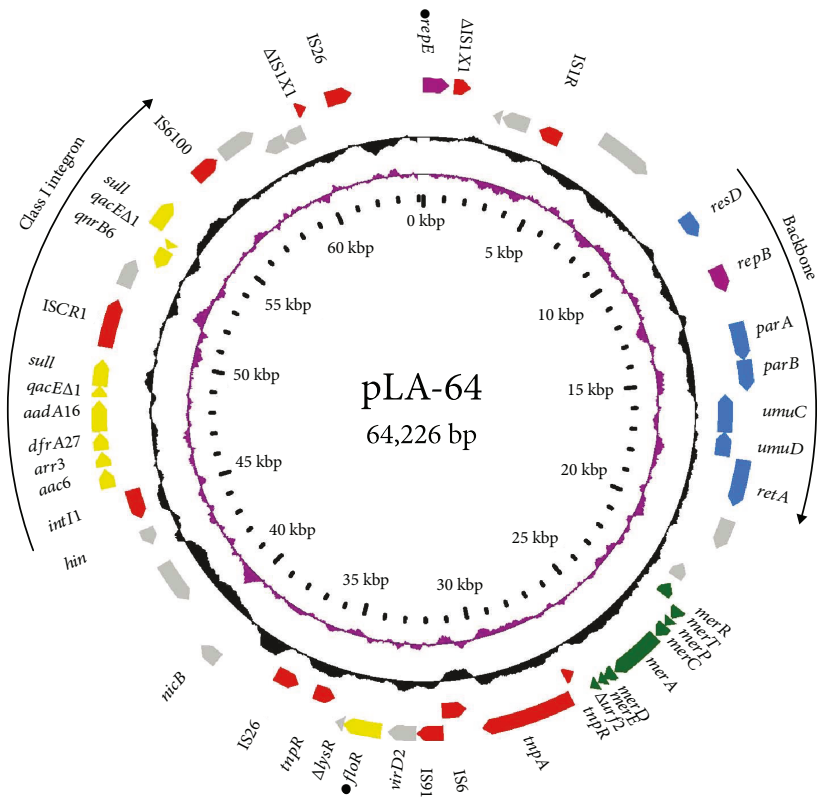

(a)

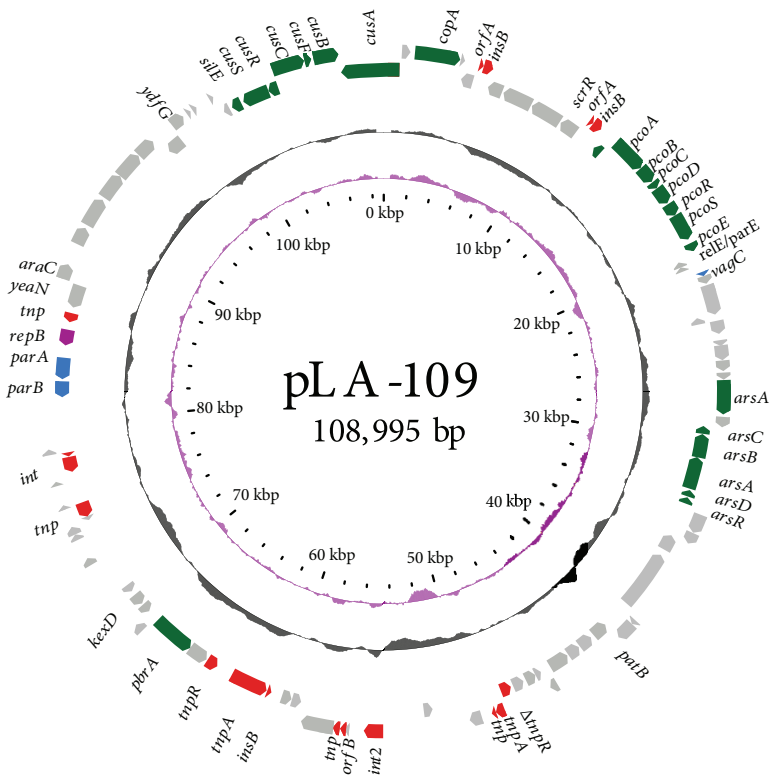

(b)

Figure 1: Genomic structure of the plasmids pLA-64 (a) and pLA-109 (b). Genes are denoted by arrows and are colored based on gene function classification. Counting from the outside toward the center: (1) genes encoded on the leading strand (outwards) or the lagging strand (inwards) with the hypothetical protein left blank; (2) an average $\mathrm{G}+\mathrm{C}$ content of $50 \%$, whereas a $\mathrm{G}+\mathrm{C}$ content of more than $50 \%$ is shown toward the outside and a G+C content of less than $50 \%$ toward the inside; (3) GC skew (G-C/G+C) with a positive GC skew toward the outside and a negative GC skew toward the inside; and (4) scale in bp. Genes with different functions are shown in different colors: red: transposable elements; yellow: drug resistance; green: heavy metal resistance; blue: backbone; purple: replication; gray: genes with other functions.

or the resistance mechanism of $L$. adecarboxylata to florfenicol. Thus, to elucidate the florfenicol resistance mechanism of $L$. adecarboxylata, we sequenced an $L$. adecarboxylata isolate designated as R25 isolated from rabbit feces with high MICs to florfenicol $(128 \mu \mathrm{g} / \mathrm{mL})$ and chloramphenicol $(128 \mu \mathrm{g} / \mathrm{mL})$ (Table 4$)$. Although three complete genomes of the same species of $L$. adecarboxylata are available in the NCBI nucleotide database, no floR gene was identified among them. Of these three L. adecarboxylata isolates, only one, USDA-ARS-USMARC-60222 (CP013990.1), without a plasmid was from an animal (calf). The other two, LSNIH3 (CP026387.1) and LSNIH1 (CP026167.1), were from the hospital environment of housekeeping closet drains in the United States.

The genome of $L$. adecarboxylata R25 consists of a $4.74 \mathrm{Mb}$ circular chromosome encoding 4,293 open reading frames (ORFs) and two plasmids, pLA-64 (64,226 bp) and pLA-109 (108,995bp) encoding 82 and 121 ORFs, respectively (Figures 1 (a) and 1 (b) and Table 5). Comparative genomic analysis showed that the genomes of the three L. adecarboxylata strains (USDA-ARS-USMARC60222, CP013990.1; LSNIH3, CP026387.1; and LSNIH1, 
TABLE 5: General features of the L. adecarboxylata R25 genome.

\begin{tabular}{lccc}
\hline & Chromosome & pLA-64 & pLA-109 \\
\hline Size (bp) & $4,741,546$ & 64,226 & 108,995 \\
GC content (\%) & 56.43 & 53.04 & 50.14 \\
Open reading frames (ORFs) & 4,293 & 82 & 121 \\
Known proteins & $3355(79.2 \%)$ & $50(62.5 \%)$ & $79(70.5 \%)$ \\
Hypothetical proteins & $882(20.8 \%)$ & $30(37.5 \%)$ & $33(29.5 \%)$ \\
Protein coding & $87.31 \%$ & $80.43 \%$ & $77.95 \%$ \\
Average ORF length (bp) & 958 & 645 & 758 \\
Average protein length (aa) & 977 & 214 & 251 \\
tRNAs & 85 & 0 & 0 \\
rRNA operons & $7 *(16 s-23 s-5 s)$ & 0 & 0 \\
\hline
\end{tabular}

CP026167.1) share the highest chromosome sequence identities with that of $L$. adecarboxylata R25, at $82 \%$, $81 \%$, and $81 \%$ coverage and $94 \%, 93 \%$, and $92 \%$ identity, respectively.

Annotation of the complete genome of L. adecarboxylata R25 revealed that it encodes nine drug resistance genes, conferring resistance to chloramphenicols (floR and $m d f A$ ), aminoglycosides ( $a a c\left(6^{\prime}\right)-I b-c r$ and aadA16), quinolones ( $q n r B 6)$, sulfonamides (sul1), trimethoprim (dfrA27), rifampicin (arr-3), and a quaternary ammonium compound ( $q a c E \Delta 1)$. Five resistance genes (floR, aac $\left(6^{\prime}\right)-I b-c r$, arr-3, aadA16, and qnrB6) with their promoter regions in $L$. adecarboxylata R25 were cloned, and expression of these resistance genes conferred resistance to the corresponding antibiotics in comparison with the MIC levels of the control strain $\mathrm{DH} 5 \alpha$. Expression of the floR gene resulted in a 3-fold increase in resistance to florfenicol, with the $\operatorname{aac}\left(6^{\prime}\right)-I b-c r$ and aadA16 genes conferring at least 3- to 6 -fold increase in resistance to amikacin, kanamycin, streptomycin, and spectinomycin. For the qnrB6 gene expression, at least a 2 -fold increase in resistance to nalidixic acid, norfloxacin, and ciprofloxacin was achieved and a 4fold increase in resistance to rifampin was obtained with arr-3 (Table 4). The resistance gene profile and MIC results for the cloned resistance genes against florfenicol, chloramphenicol, rifampin, and spectinomycin were in accordance with the host's resistance phenotypes (Table 4). A few publications have demonstrated that in nonclinical $L$. adecarboxylata isolates, several resistance genes (such as $r m t B, q n r A, O q x b$, and $q e p$ ) mediate resistance to certain antibiotics, including aminoglycosides and fluoroquinolone $[17,31]$. Resistance to beta-lactams among clinical L. adecarboxylata isolates can be ascribed to the acquisition of resistance plasmids carrying $b l a_{\mathrm{SHV}-12}$ and $b l a_{\mathrm{NDM}-1}$ [32], but these isolates are generally sensitive to commonly used antibiotics such as tetracyclines, aminoglycosides, quinolones, and chloramphenicol [33]. The floR, aadA16, and qnrB6 genes identified in L. adecarboxylata R25 have not been reported previously.

3.4. Comparative Analysis of the Resistance Plasmid pLA-64 and Resistance Gene-Related Sequences. Among 9 resistance genes, only 1 ( $m d f A)$ is located on the chromosome, whereas the other 8 (floR, $\operatorname{aac}\left(6^{\prime}\right)-I b-c r, \operatorname{arr}-3, d f r A 27, \operatorname{aad} A 16$, $q a c E \Delta 1$, sul1, and $q n r B 6)$ are encoded by the plasmid pLA64 (Figure 1(a)). In addition to drug resistance genes, the complete sequence encodes four clusters of heavy metal resistance genes, with one mercury resistance gene cluster on pLA-64 (Figure 1(a)) and the other three (copper, copper/silver, and arsenate resistance gene clusters) on plasmid pLA109 (Figure 1(b)).

The sequences sharing the greatest similarities to pLA-64 are the three plasmids p02085-tetA (MH477637.1) of $C$. freundii strain 1509-02085 (no original information) and p234 (CP021163.1) and p388 (CP021168.1) from E. cloacae strains isolated from humans. These plasmids encompass nearly the entire sequence of pLA-64, with more than $99 \%$ coverage and $99 \%$ identity. p02085-tetA is $68 \mathrm{~kb}$ in length and p234 $69 \mathrm{~kb}$, only 4 and $5 \mathrm{~kb}$ longer than pLA-64, respectively, with an extra resistance gene-related fragment (encoding tetR-tetD-frmA-frmB-IS26) inserted at position $37 \mathrm{~kb}$ of pLA-64. p388 is $79 \mathrm{~kb}$ in size and $15 \mathrm{~kb}$ longer than pLA64 , with two extra resistance gene-related fragments inserted at positions $37 \mathrm{~kb}$ and $51 \mathrm{~kb}$ of pLA-64, respectively. The plasmid with low similarity to pLA-64 is plasmid1 (CP009116.1) from a human clinical K. pneumoniae strain; it is $95 \mathrm{~kb}$ in length ( $31 \mathrm{~kb}$ longer than pLA-64) and contains $81 \%(52 / 64)$ of the sequence of pLA-64 but without the floR gene-related region (encoding IS6- $\Delta \mathrm{IS} 91$-virD2-floR- $\Delta l y s R$ ) (Figure 2). All these plasmids belong to the same Inc group carrying two replicons: FIA(HI1) and R. However, no plasmids from the two Leclercia strains LSNIH3 (CP026387.1) and LSNIH1 (CP026167.1) share sequence identity of more than $28 \%$ with pLA-64. The plasmids in these two strains harbor the replicons of other Inc groups, including N, FII (pCTU2), HI1A (CIT), and HI1B (CIT), as opposed to the FIA (HI1) and R of pLA-64. These findings indicate that pLA-64 homologous plasmids may transfer among bacteria of different genera of various (animal and human) origins.

The plasmid pLA-64 consists roughly of two parts: a backbone and a variable region. The backbone is composed of segments responsible for replication (repBE), DNA repair $(u m u C D)$, and plasmid maintenance (parAB), 


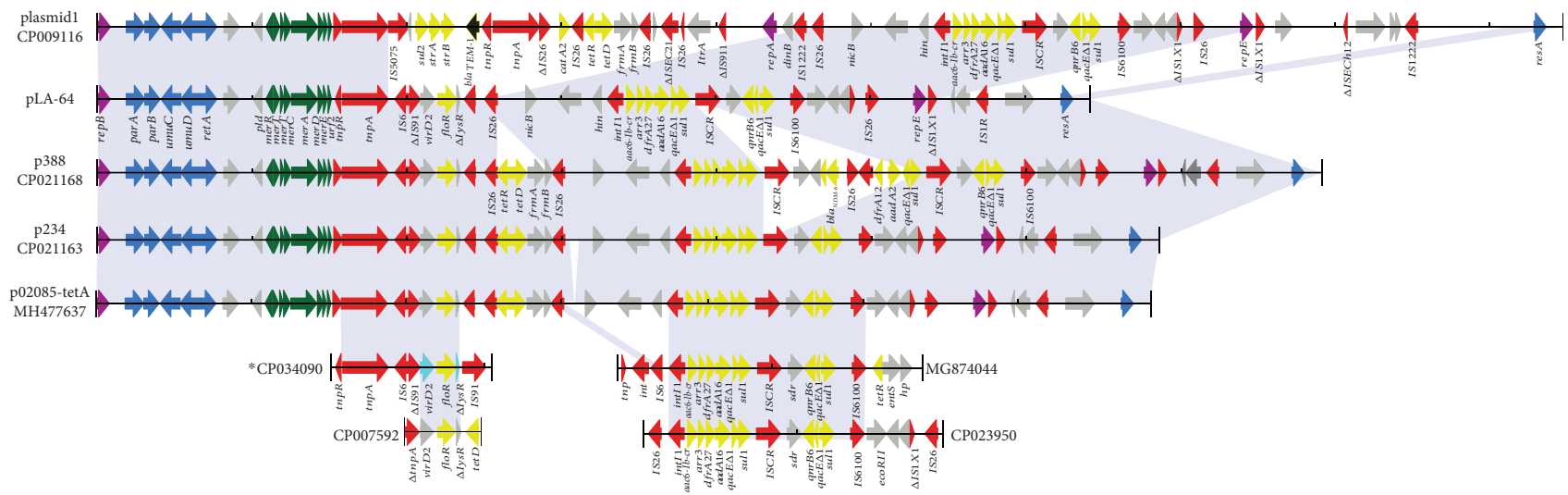

Figure 2: Comparative genomic analysis of pLA-64 and homologous sequences from other plasmids. plasmid1 (CP009116.1), from a K. pneumoniae strain isolated from the human; p388 (CP021168.1), from E. cloacae strain 388 isolated from the human; p234 (CP021163.1), from E. cloacae strain 234 isolated from the human; p02085-tetA (MH477637.1), from C. freundii strain 1509-02085 without origin information; CP034090.1, the chromosome of P. mirabilis strain PmSC1111 isolated from swine; CP007592.1, the chromosome of E. coli O157:H16 Santai isolated from the duck; MG874044.1, plasmid pSa76-CIP of Salmonella sp. without origin information; and CP023950.1, plasmid unnamed3 of K. pneumoniae FDAARGOS_447 isolated from the human. Genes with different functions are shown in different colors: red: transposable elements; yellow: drug resistance; green: heavy metal resistance; blue: backbone; purple: replication; gray: genes with other functions.

whereas the variable regions harbor a number of MGEs, such as insertion sequences, transposons, and an integron. All eight resistance genes encoded by the pLA-64 are related to MGEs. Six are found in a class 1 integron (intl1-aac $\left(6^{\prime}\right)$-Ib-cr-arr-3-dfrA27-aadA16-qacED1-sul1); the other two ( $q n r B 6$ and floR) are related to transposons. The flo $R$ gene is located in a fragment approximately $7.6 \mathrm{~kb}$ in length encoding the Tn3-IS6- $\Delta$ IS91-virD2-floR$\triangle l y s R$ gene cluster (Figures $1(\mathrm{a})$ and 2). In addition to the plasmids (p02085-tetA, p234, p388, and plasmid1) mentioned above, sequences with higher identities with resistance gene-related MGEs were identified in other plasmid or chromosome sequences, such as those encoded by the chromosome of Proteus mirabilis strain PmSC1111 (CP034090.1), the chromosome of E. coli O157:H16 Santai (CP007592.1), Salmonella sp. plasmid pSa76-CIP (MG874044.1), and plasmid unnamed3 of K. pneumoniae FDAARGOS_447 (CP023950.1) (Figure 2). The gene array of the class 1 integron has also been identified in other animal bacteria of different species, such as E. coli and Enterobacter amnigenus isolated from swine feces and swine farm wastewater, respectively [34]. Similarly, the qnrB6-related gene cluster was identified on K. pneumoniae plasmid pLC24 isolated from dog vomit [35]. Although the genetic environment of the floR gene encoded on the chromosome or plasmid from different bacteria differed, the gene cluster of virD2-floR- $\Delta l y s R$ encoded on pLA-64 in this work is conserved in most cases [30].

\section{Conclusion}

The results of this work show high rates of resistance to florfenicol (61.6\%, 180/292) and chloramphenicol (65.1\%, $190 / 292$ ) among animal Enterobacteriaceae isolates. The floR gene is common among various species (64.4\%. 188/292), though $c f r$ was only identified in some Proteus spp. $(1.0 \%$,
3/292). All resistance genes including floR encoded on the plasmid pLA-64 in L. adecarboxylata R25 are related to MGEs. Comparative genomic analysis demonstrated that the sequences sharing the greatest similarities to pLA-64 are three plasmids from $C$. freundii and E. cloacae strains isolated from humans. These findings indicate a high rate of florfenicol resistance among local animal bacteria, with the floR gene also being highly prevalent. Resistance plasmids may be transferred between bacteria of different species or genera and of different (animal and human) origins and cause resistance dissemination.

4.1. Accession Numbers. The complete nucleotide sequences of the chromosome and plasmids have been submitted to the NCBI database, and the accession numbers of chromosome, pLA-64, and pLA-109 are CP035382.1, CP035381.1, and CP035380.1, respectively.

\section{Data Availability}

The data used to support the findings of this study are available from the corresponding author upon request.

\section{Conflicts of Interest}

The authors declare no conflict of interest.

\section{Authors' Contributions}

Yuanyuan Ying and Fei Wu contributed equally to this work.

\section{Acknowledgments}

This work was supported by grants from the Natural Science Foundation of Zhejiang Province (LY19C060002 and LQ17H190001), the Soft Science Project of the Lishui Science and Technology Bureau (2017RKX14), the National Natural 
Science Foundation of China (81401702, 81501808, and 81501780 ), and the Science \& Technology Project of the Inner Mongolia Autonomous Region, China (201802125).

\section{Supplementary Materials}

Table S1: distribution of Enterobacteriaceae isolated from the animal feces specimens from the animal farms in South China. (Supplementary Materials)

\section{References}

[1] I. Chantziaras, F. Boyen, B. Callens, and J. Dewulf, "Correlation between veterinary antimicrobial use and antimicrobial resistance in food-producing animals: a report on seven countries," The Journal of Antimicrobial Chemotherapy, vol. 69, no. 3, pp. 827-834, 2014.

[2] A. de Jong, V. Thomas, S. Simjee et al., "Antimicrobial susceptibility monitoring of respiratory tract pathogens isolated from diseased cattle and pigs across Europe: the VetPath study," Veterinary Microbiology, vol. 172, no. 1-2, pp. 202$215,2014$.

[3] S. Schwarz, C. Kehrenberg, B. Doublet, and A. Cloeckaert, "Molecular basis of bacterial resistance to chloramphenicol and florfenicol," FEMS Microbiology Reviews, vol. 28, no. 5, pp. 519-542, 2004.

[4] H. Liu, Y. Wang, C. Wu et al., "A novel phenicol exporter gene, $f e x B$, found in enterococci of animal origin," The Journal of Antimicrobial Chemotherapy, vol. 67, no. 2, pp. 322325, 2012.

[5] Y. Wang, Y. Lv, J. Cai et al., "A novel gene, optrA, that confers transferable resistance to oxazolidinones and phenicols and its presence in Enterococcus faecalis and Enterococcus faecium of human and animal origin," The Journal of Antimicrobial Chemotherapy, vol. 70, no. 8, pp. 2182-2190, 2015.

[6] A. Zhang, Y. Yang, H. Wang et al., "Prevalence of sulfonamide and florfenicol resistance genes in Escherichia Coli isolated from yaks (Bos Grunniens) and herdsmen in the Tibetan pasture," Journal of Wildlife Diseases, vol. 51, no. 3, pp. 626-633, 2015.

[7] A. Alessiani, L. Sacchini, E. Pontieri, J. Gavini, and E. Di Giannatale, "Molecular typing of Salmonella enterica subspecies enterica serovar Typhimurium isolated in Abruzzo region (Italy) from 2008 to 2010," Veterinaria Italiana, vol. 50, no. 1, pp. 31-39, 2014.

[8] T. He, J. Shen, S. Schwarz, C. Wu, and Y. Wang, "Characterization of a genomic island in Stenotrophomonas maltophilia that carries a novel floR gene variant," The Journal of Antimicrobial Chemotherapy, vol. 70, no. 4, pp. 1031-1036, 2015.

[9] Y. Tang, L. Dai, O. Sahin, Z. Wu, M. Liu, and Q. Zhang, "Emergence of a plasmid-borne multidrug resistance gene $c f r(\mathrm{C})$ in foodborne pathogen Campylobacter," The Journal of Antimicrobial Chemotherapy, vol. 72, no. 6, pp. 1581-1588, 2017.

[10] S. K. Chang, D. Y. Lo, H. W. Wei, and H. C. Kuo, “Antimicrobial resistance of Escherichia coli isolates from canine urinary tract infections," Journal of Veterinary Medical Science, vol. 77, no. 1, pp. 59-65, 2015.

[11] J. Lu, J. Zhang, L. Xu et al., "Spread of the florfenicol resistance floR gene among clinical Klebsiella pneumoniae isolates in China," Antimicrobial Resistance \& Infection Control, vol. 7, no. 1, p. $127,2018$.
[12] Y. Lu, H. Zhao, J. Sun et al., "Characterization of multidrugresistant Salmonella enterica serovars Indiana and Enteritidis from chickens in Eastern China," PLoS One, vol. 9, no. 5, article e96050, 2014.

[13] A. von Altrock, U. Roesler, R. Merle, and K. H. Waldmann, "Prevalence of pathogenic Yersinia enterocolitica strains on liver surfaces of pigs and their antimicrobial susceptibility," Journal of Food Protection, vol. 73, no. 9, pp. 1680-1683, 2010.

[14] Y. Wang, Y. Wang, C. M. Wu et al., "Detection of the staphylococcal multiresistance gene $c f r$ in Proteus vulgaris of food animal origin," The Journal of Antimicrobial Chemotherapy, vol. 66, no. 11, pp. 2521-2526, 2011.

[15] H. Leclerc, "Biochemical study of pigmented Enterobacteriaceae," Annales de l'Institut Pasteur, vol. 102, pp. 726-741, 1962.

[16] K. Tamura, R. Sakazaki, Y. Kosako, and E. Yoshizaki, "Leclercia adecarboxylata Gen. Nov., Comb. Nov., formerly known as Escherichia adecarboxylata," Current Microbiology, vol. 13, no. 4, pp. 179-184, 1986.

[17] Q. Yao, Z. Zeng, J. Hou et al., "Dissemination of the $r m t B$ gene carried on IncF and IncN plasmids among Enterobacteriaceae in a pig farm and its environment," The Journal of Antimicrobial Chemotherapy, vol. 66, no. 11, pp. 2475-2479, 2011.

[18] A. De Mauri, D. Chiarinotti, S. Andreoni, G. L. Molinari, N. Conti, and M. De Leo, "Leclercia adecarboxylata and catheter-related bacteraemia: review of the literature and outcome with regard to catheters and patients," Journal of Medical Microbiology, vol. 62, Part_10, pp. 1620-1623, 2013.

[19] S. Koren, B. P. Walenz, K. Berlin, J. R. Miller, N. H. Bergman, and A. M. Phillippy, "Canu: scalable and accurate long-read assembly via adaptive $k$-mer weighting and repeat separation," Genome Research, vol. 27, no. 5, pp. 722-736, 2017.

[20] A. Petkau, M. Stuart-Edwards, P. Stothard, and G. Van Domselaar, "Interactive microbial genome visualization with GView," Bioinformatics, vol. 26, no. 24, pp. 3125-3126, 2010.

[21] C. Kehrenberg, J. Mumme, J. Wallmann et al., "Monitoring of florfenicol susceptibility among bovine and porcine respiratory tract pathogens collected in Germany during the years 2002 and 2003," The Journal of Antimicrobial Chemotherapy, vol. 54, no. 2, pp. 572-574, 2004.

[22] D. A. E. Dayao, J. S. Gibson, P. J. Blackall, and C. Turni, “Antimicrobial resistance in bacteria associated with porcine respiratory disease in Australia," Veterinary Microbiology, vol. 171, no. 1-2, pp. 232-235, 2014.

[23] Y. Zhu, H. Lai, L. Zou et al., "Antimicrobial resistance and resistance genes in Salmonella strains isolated from broiler chickens along the slaughtering process in China," International Journal of Food Microbiology, vol. 259, pp. 43-51, 2017.

[24] M. G. Maaland, S. S. Mo, S. Schwarz, and L. Guardabassi, "In vitro assessment of chloramphenicol and florfenicol as second-line antimicrobial agents in dogs," Journal of Veterinary Pharmacology and Therapeutics, vol. 38, no. 5, pp. 443450, 2015.

[25] M. Ture, I. Altinok, and H. Alp, "Effects of cage farming on antimicrobial and heavy metal resistance of Escherichia coli, Enterococcus faecium, and Lactococcus garvieae," Microbial Drug Resistance, vol. 24, no. 9, pp. 1422-1430, 2018.

[26] A. Cloeckaert, S. Baucheron, and E. Chaslus-Dancla, "Nonenzymatic chloramphenicol resistance mediated by IncC plasmid R55 is encoded by a floR gene variant," Antimicrobial Agents and Chemotherapy, vol. 45, no. 8, pp. 2381-2382, 2001. 
[27] F. Rahmani, A. Fooladi, S. Marashi, and M. Nourani, "Drug resistance in Vibrio cholerae strains isolated from clinical specimens," Acta Microbiologica et Immunologica Hungarica, vol. 59, no. 1, pp. 77-84, 2012.

[28] Y. Wang, W. Zhang, J. Wang et al., "Distribution of the multidrug resistance gene cfr in Staphylococcus species isolates from swine farms in China," Antimicrobial Agents and Chemotherapy, vol. 56, no. 3, pp. 1485-1490, 2012.

[29] M. A. Arcangioli, S. Leroy-Sétrin, J. L. Martel, and E. ChaslusDancla, "A new chloramphenicol and florfenicol resistance gene flanked by two integron structures in Salmonella typhimurium DT104," FEMS Microbiology Letters, vol. 174, no. 2, pp. 327-332, 1999.

[30] A. Cloeckaert, S. Baucheron, G. Flaujac et al., "Plasmidmediated florfenicol resistance encoded by the floR gene in Escherichia coli isolated from cattle," Antimicrobial Agents and Chemotherapy, vol. 44, no. 10, pp. 2858-2860, 2000.

[31] O. R. Akinbami, S. Olofinsae, and F. A. Ayeni, "Prevalence of extended spectrum beta lactamase and plasmid mediated quinolone resistant genes in strains of Klebsiella pneumonia, Morganella morganii, Leclercia adecarboxylata and Citrobacter freundii isolated from poultry in South Western Nigeria," PeerJ, vol. 6, article e5053, 2018.

[32] F. Sun, Z. Yin, J. Feng et al., "Production of plasmid-encoding NDM-1 in clinical Raoultella ornithinolytica and Leclercia adecarboxylata from China," Frontiers in Microbiology, vol. 6, p. $458,2015$.

[33] I. Stock, S. Burak, and B. Wiedemann, "Natural antimicrobial susceptibility patterns and biochemical profiles of Leclercia adecarboxylata strains," Clinical Microbiology and Infection, vol. 10, no. 8, pp. 724-733, 2004.

[34] Z. Liu, Z. Zhang, H. Yan, J. Li, and L. Shi, "Isolation and molecular characterization of multidrug-resistant Enterobacteriaceae strains from pork and environmental samples in Xiamen, China," Journal of Food Protection, vol. 78, no. 1, pp. 78-88, 2015.

[35] J. Ma, Z. Zeng, Z. Chen et al., "High prevalence of plasmidmediated quinolone resistance determinants qnr, aac $\left(6^{\prime}\right)-I b$ $c r$, and qepA among ceftiofur-resistant Enterobacteriaceae isolates from companion and food-producing animals," Antimicrobial Agents and Chemotherapy, vol. 53, no. 2, pp. 519524, 2009. 


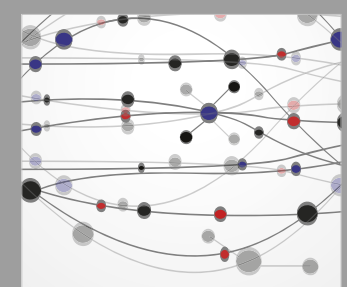

The Scientific World Journal
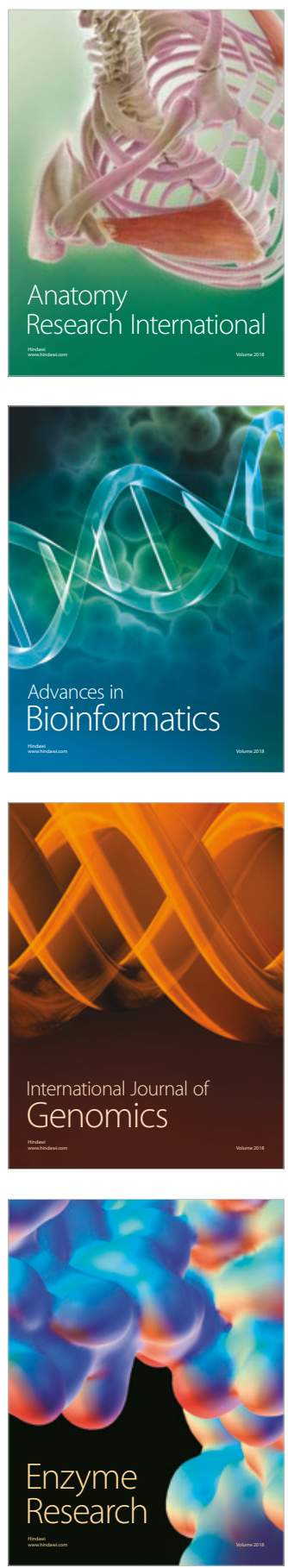
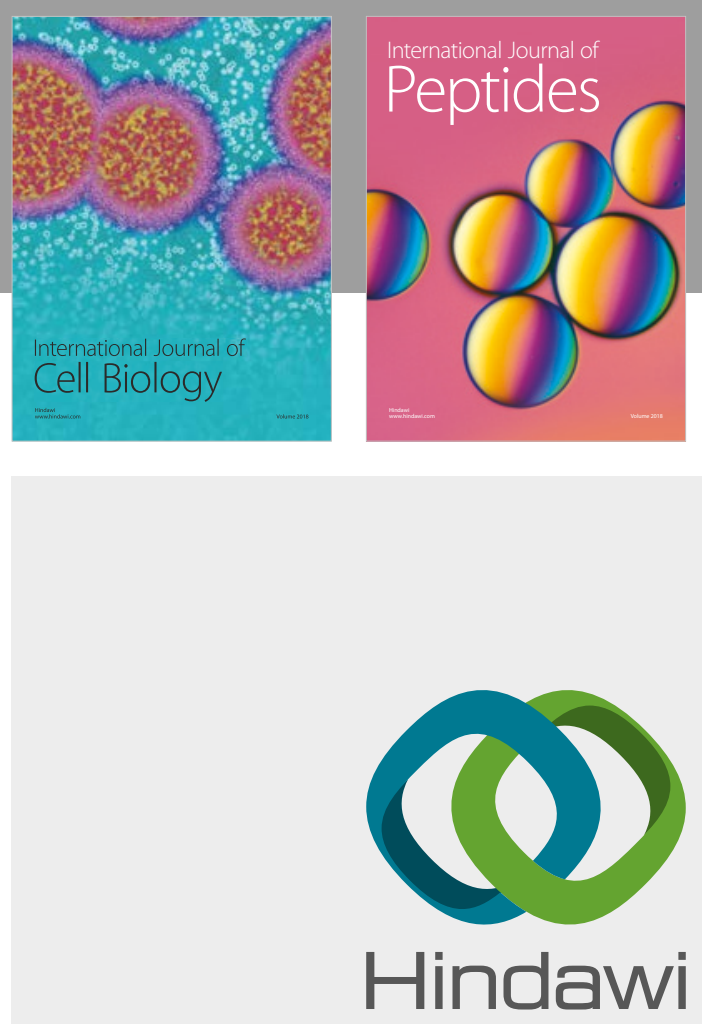

Submit your manuscripts at

www.hindawi.com
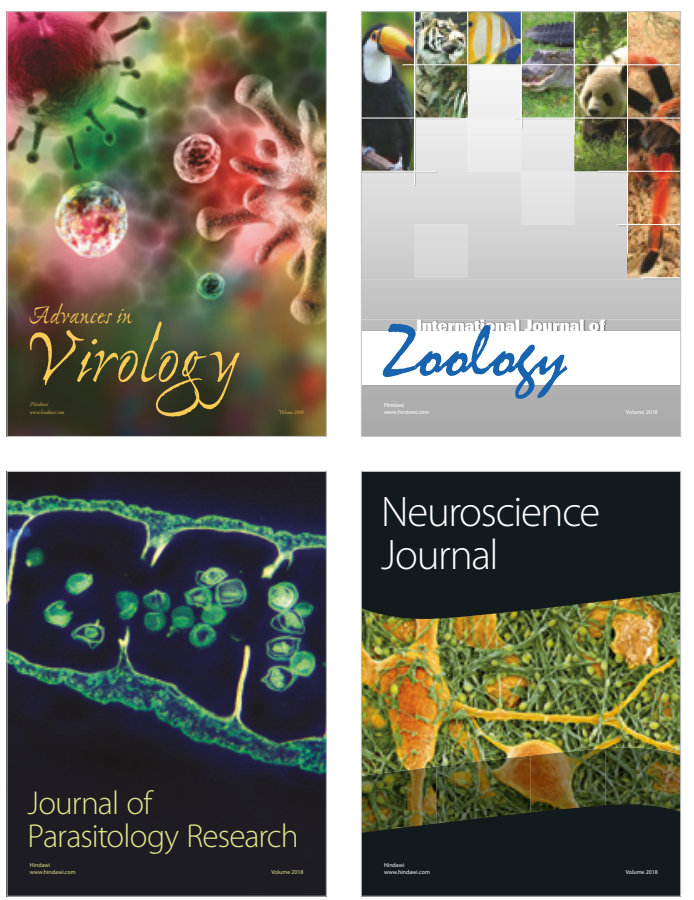
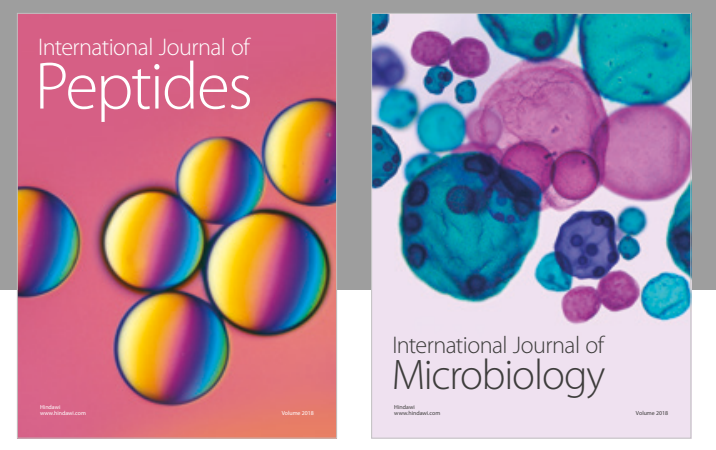

nternational Journal of Microbiology
Journal of
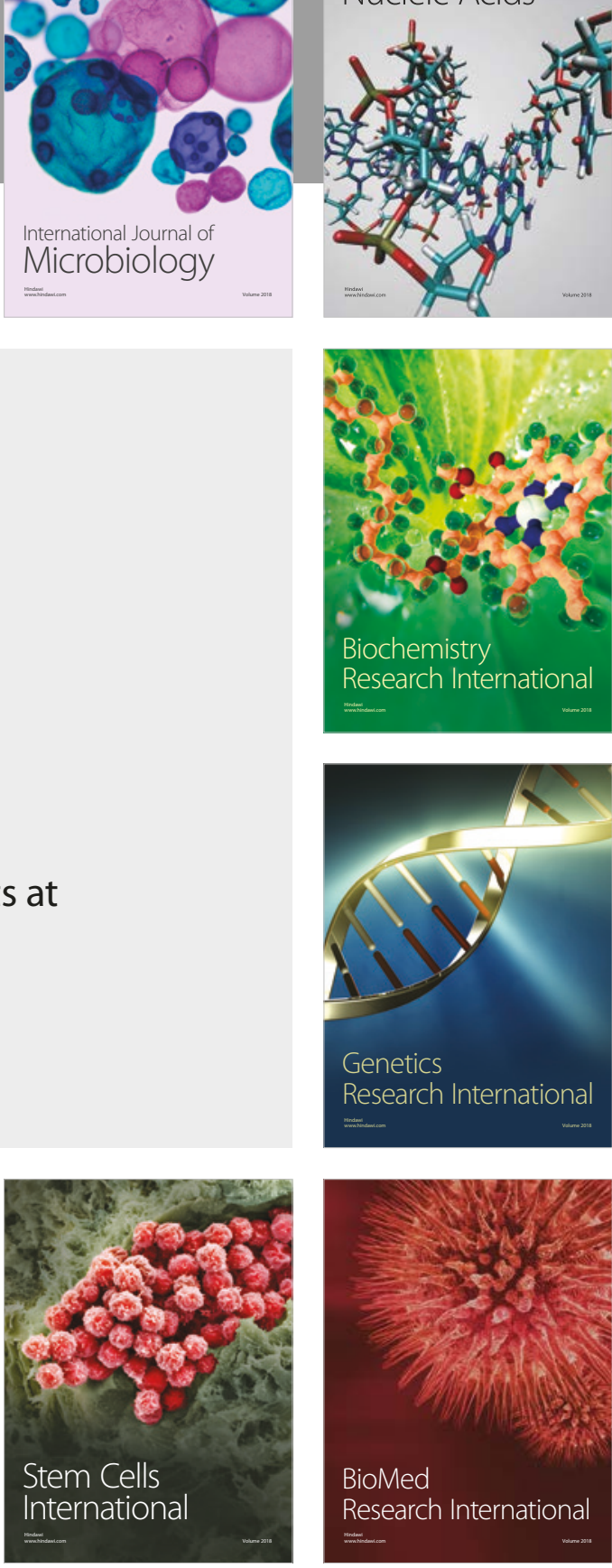
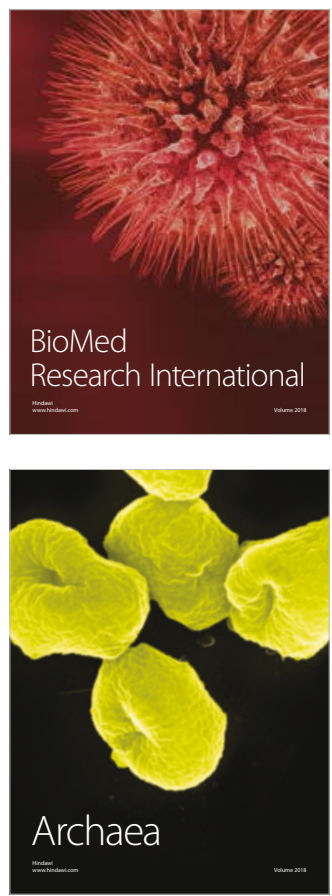